\title{
Presión hidrostática
}

\section{Hydrostatic pressure}

\section{Martín Ortiz-Domínguez ${ }^{a}$, Arturo Cruz-Avilés ${ }^{b}$, Jorge Zuno-Silva ${ }^{c}$, Carlos E. Borja-Soto ${ }^{d}$, Cesar Mendoza-Gómora ${ }^{e}$}

\begin{abstract}
:
Hydrostatics is used to determine the forces acting on floating or submerged bodies and the forces generated by some devices such as hydraulic dams, liquid storage tanks, dikes and gates. In this work, the hydrostatic forces acting on fully submerged or partially submerged surfaces are determined by applying fluid statics.
\end{abstract}

Keywords:

Hydrostatic force, fluid force, pressure, fluid static

\section{Resumen:}

La hidrostática se utiliza para determinar las fuerzas que actúan sobre cuerpos flotantes o sumergidos y las fuerzas que generan algunos dispositivos como las presas hidráulicas, tanques de almacenamiento de líquidos, diques y compuertas. En el presente trabajo se determinan las fuerzas hidrostáticas que actúan sobre las superficies totalmente sumergidas o parcialmente sumergidas aplicando la estática de fluidos.

Palabras Clave:

Fuerza hidrostática, fuerza del fluido, presión, estática de fluidos

\section{Introducción}

Las fuerzas hidrostáticas son las fuerzas resultantes causadas por la carga de presión de un líquido que actúa sobre superficies sumergidas (ver Figura 1). Esta fuerza hidrostática no es la misma que la fuerza del fluido en el fondo de la presa o del canal, ya que se aplica en un ángulo más pronunciado (normalmente ángulo recto) y cambia con la altura del agua (inmersión del plano). Los ingenieros necesitan saber cómo predecir esta fuerza para ayudar a entender la fuerza y el diseño de las estructuras necesarias para resistirla. 1-10

\footnotetext{
a Autor de Correspondencia, Licenciatura en Ingeniería Mecánica, Escuela Superior de Ciudad Sahagún, UAEH, Tepeapulco, Hidalgo, México, ORCID: 0000-0003-4475-9804,Email: martin_ortiz@uaeh.edu.mx;

b Licenciatura en Ingeniería Mecánica, Escuela Superior de Ciudad Sahagún, UAEH, Tepeapulco, Hidalgo, México, ORCID: 0000-00030455-1646, Email: arturo_cruz8085@uaeh.edu.mx;

c Licenciatura en Ingeniería Mecánica, Escuela Superior de Ciudad Sahagún, UAEH, Tepeapulco, Hidalgo, México, https://orcid.org/00000002-1997-5399,Email: jorge_zuno@uaeh.edu.mx;

d Licenciatura en Ingeniería Mecánica, Escuela Superior de Ciudad Sahagún, UAEH, Tepeapulco, Hidalgo, México, https://orcid.org/00000003-3385-8348. Email: carlos_borja@uaeh.edu.mx;

e Licenciatura en Ingeniería Mecánica, Escuela Superior de Ciudad Sahagún, UAEH, Tepeapulco, Hidalgo, México,

https://orcid.org/0000-0003-1462-0588.Email: cesar_mendoza@uaeh.edu.mx;
} 


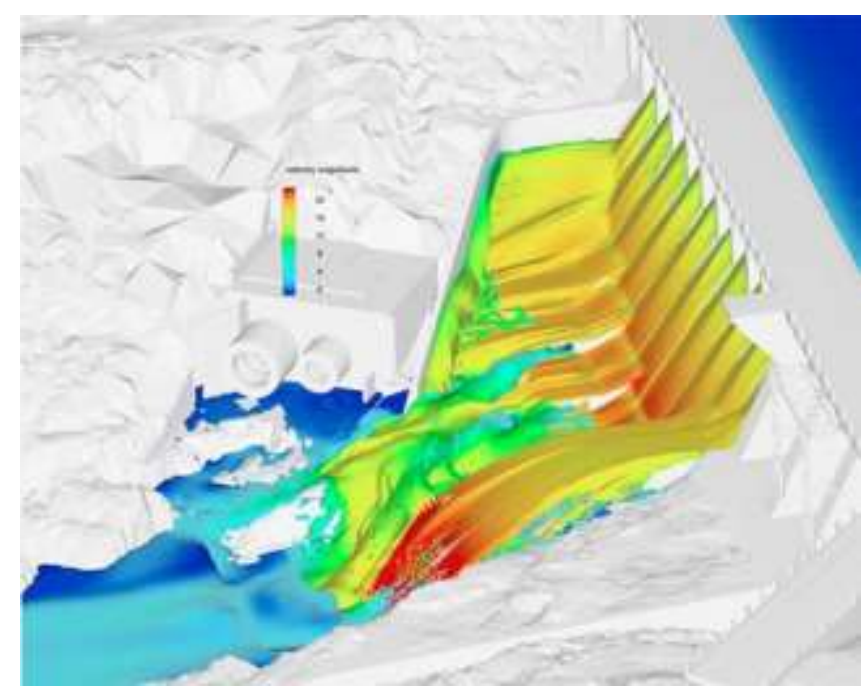

Figura 1. Presión hidrostática que actúa sobre las superficies sumergidas.

Fuente: Elaboración propia.

\section{Objetivo general}

Estudiar la fuerza hidrostática en una superficie plana vertical total o parcialmente sumergida, a través de la definición fundamental de presión y hacer una comparación entre ambas configuraciones de llenado.

\section{Objetivos específicos}

- Estudiar el momento debido a la fuerza hidrostática en una superficie plana vertical total o parcialmente sumergida, a través de la suma de momentos sobre el punto de pivote 0 y hacer una comparación entre ambas configuraciones de llenado.

- Encontrar el punto (ubicación) de la fuerza hidrostática, a través de las magnitudes geométricas del equipo empleado, para hacer una comparación entre ambas configuraciones de llenado.

\section{Aplicaciones prácticas}

La ubicación y la magnitud de la fuerza de presión del agua que actúa sobre las estructuras de control del agua, como presas (ver Figura 1), contenedores de líquidos (ver Figura 2), diques y compuertas (Figura 3), son muy importantes para su diseño estructural. La fuerza hidrostática y su línea de acción también son necesarias para el diseño de muchas partes del equipo hidráulico.

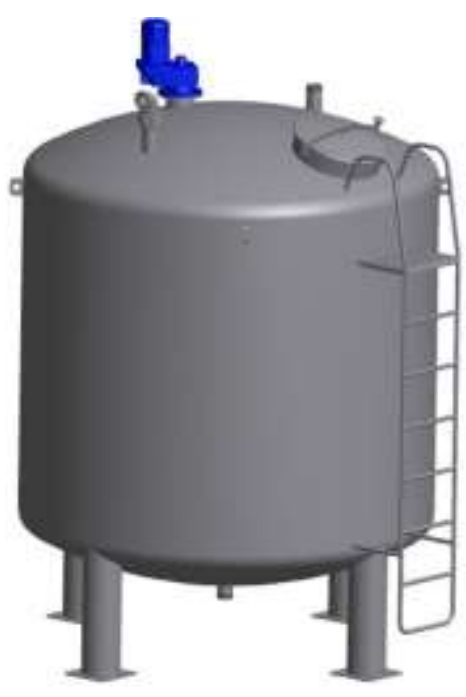

Figura 2. Contenedor de líquidos, el fluido ejerce una presión hidrostática sobre el fondo y las paredes del contenedor.

Fuente: Elaboración propia.

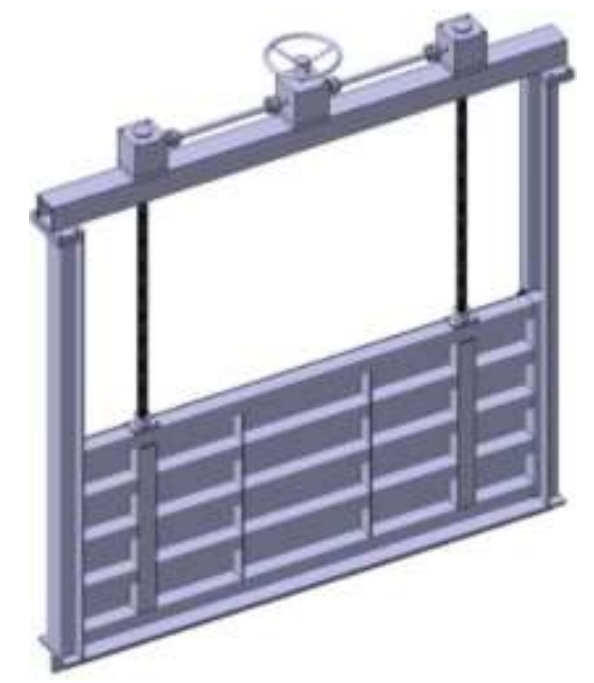

Figura 3. Compuerta para canal, cuyo fin es controlar, detener y regular el flujo de agua.

Fuente: Elaboración propia.

\section{Teoría}


El centro de presión puede definirse como "el punto en un plano en el que se puede decir que el empuje total de fluido actúa normalmente en ese plano". Como se muestra en la Figura 4, la presión hidrostática ejercida por un líquido de densidad $\rho$ o peso específico $\gamma$, a una profundidad $h$ bajo la superficie, es:

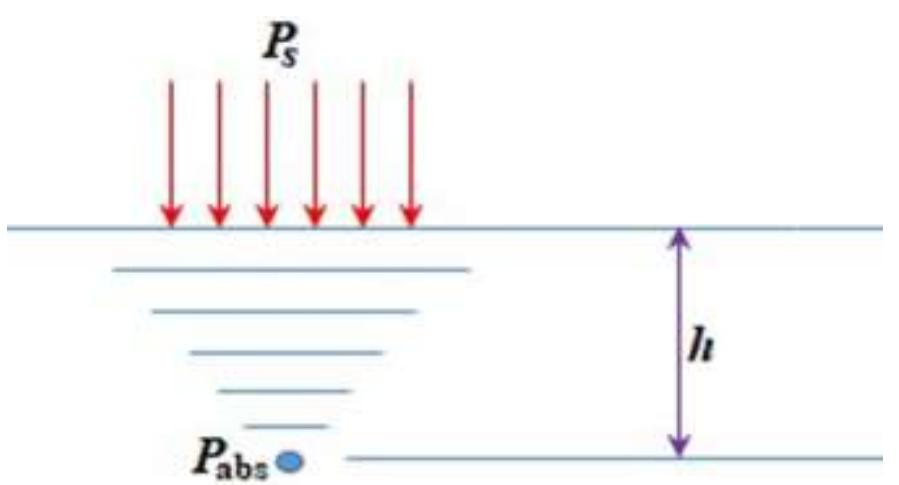

Figura 4. Esquema sobre la presión hidrostática ejercida por un fluido.

Fuente: Elaboración propia.

$$
P=\rho g h \quad(1)
$$

Esta es la presión manométrica, debida únicamente a la columna de líquido de la altura $h$. Para obtener la presión absoluta $P_{a b s}$ en la profundidad $h$, debemos añadir la presión $P_{s}$ que se aplica en la superficie del líquido, dando:

$$
P_{a b s}=P_{s}+\rho g h
$$

Considerando a la Figura (5), considere un elemento en la profundidad inicial $y$, la anchura $d y$, luego la fuerza sobre el elemento viene dada por:

$$
d F=\rho g(y \cos \theta-h) B d y
$$

La sumatoria de momentos alrededor del pivote (punto 0) está dado por:

$$
\sum M_{0}=0
$$

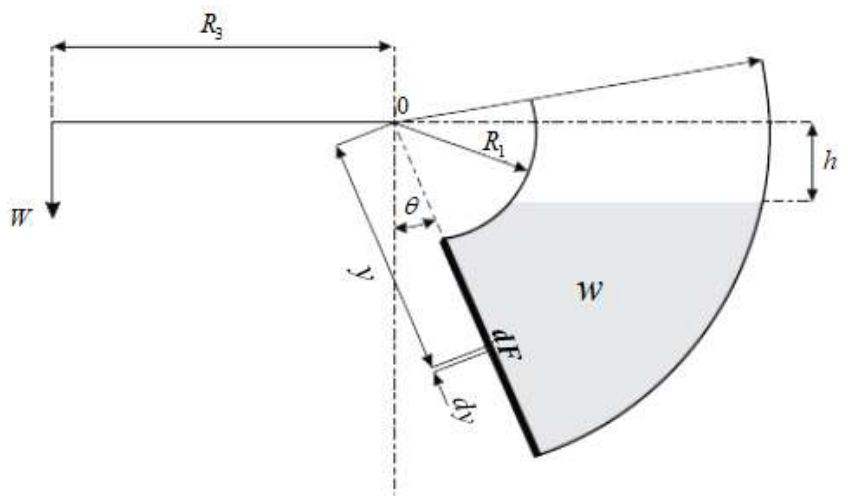

Figura 5. Esquema del centro del equipo de presión. Fuente: Elaboración propia.

De acuerdo con la Figura 5, y considerando la Ec. (4), tenemos:

$$
\begin{gathered}
W R_{3}-\rho g B \int\left(y^{2} \cos \theta-h y\right) d y=0 \\
M=W R_{3}=\rho g B \int\left(y^{2} \cos \theta-h y\right) d y
\end{gathered}
$$

\section{Caso 1: Plano totalmente sumergido $\left(h<R_{1} \cos \theta\right)$}

Cuando el plano está completamente sumergido (ver Figura 6), el agua cubre toda la superficie del área del plano y el momento aplicado debe ser inversamente proporcional a la altura del agua, así que:

\section{Límites $R_{1}$ y $R_{2}$}

Por lo tanto: 


$$
\begin{aligned}
& M=\rho g B \int_{y=R_{1}}^{y=R_{2}}\left(y^{2} \cos \theta-h y\right) d y \\
& M=\rho g B\left(\frac{\cos \theta y^{3}}{3}-\frac{h y^{2}}{2}\right)_{y=R_{1}}^{y=R_{2}}
\end{aligned}
$$

Evaluando los límites superior e inferior en la Ec. (8):

$$
M=\frac{\rho g B \cos \theta}{3}\left(R_{2}^{3}-R_{1}^{3}\right)-\frac{\rho g B h}{2}\left(R_{2}^{2}-R_{1}^{2}\right)
$$

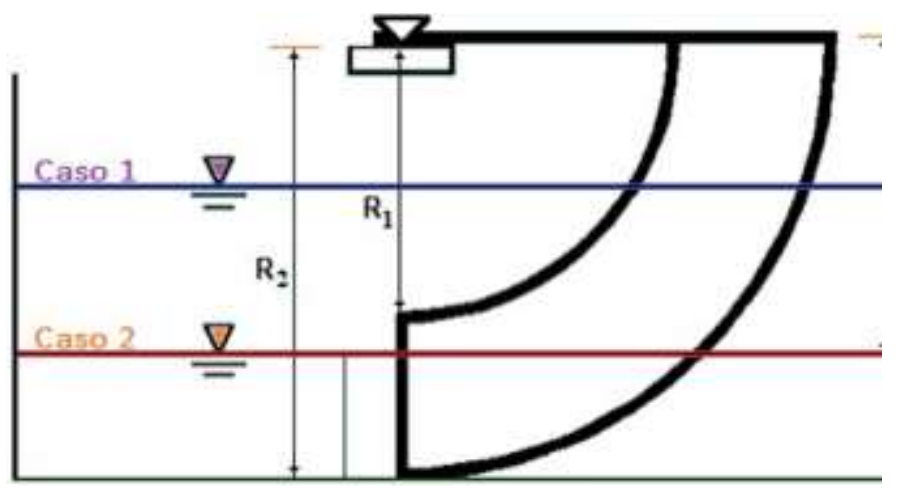

Figura 6. Esquema representativo de los dos casos del centro de presión.

Fuente: Elaboración propia.

La Ec. (9) es de la forma $y=m x+b$. Una gráfica de $M$ contra de $h$ producirá una línea recta con pendiente $-\rho g B\left(R_{2}^{2}-R_{1}^{2}\right) / 2$. De tal manera que el valor de $\rho g$ puede ahora ser determinado.

Caso 2: Plano parcialmente sumergido ( $h>R_{1} \cos \theta$ )

Cuando el plano está parcialmente sumergido (ver Figura 6), el agua sólo cubre una parte del área del plano y el momento aplicado no es proporcional a la altura del agua
. El momento en este caso viene dado por:

Límites $R_{2}$ y $h \sec \theta$

Por lo tanto:

$$
M=\rho g B \int_{y=h \sec \theta}^{y=R_{2}}\left(y^{2} \cos \theta-h y\right) d y
$$

$$
M=\rho g B\left(\frac{\cos \theta y^{3}}{3}-\frac{h y^{2}}{3}\right)_{y=h \sec \theta}^{y=R_{2}}
$$

Evaluando los límites superior e inferior en la Ec. (11):

$$
\begin{gathered}
M=\frac{\rho g B \cos \theta}{3}\left(R_{2}^{3}-h^{3} \sec ^{3} \theta\right) \\
-\frac{\rho g B h}{2}\left(R_{2}^{2}-h^{2} \sec ^{2} \theta\right) \\
M=\frac{\rho g B \cos \theta R_{2}^{3}}{3}-\frac{\rho g B \sec ^{2} \theta h^{3}}{3} \\
-\frac{\rho g B h R_{2}^{2}}{2}+\frac{\rho g B \sec ^{2} \theta h^{3}}{2}
\end{gathered}
$$

$$
\begin{aligned}
& M=\frac{\rho g B \cos \theta R_{2}^{3}}{3}-\frac{2 \rho g B \sec ^{2} \theta h^{3}}{6} \\
& -\frac{\rho g B h R_{2}^{2}}{2}+\frac{3 \rho g B \sec ^{2} \theta h^{2}}{6}
\end{aligned}
$$

Reduciendo términos en la Ec. (14):

$$
M=\frac{\rho g B \cos \theta R_{2}^{3}}{3}-\frac{\rho g B h R_{2}^{2}}{2}+\frac{\rho g B \sec ^{2} \theta h^{3}}{6}
$$

Donde:

$\rho=$ densidad del fluido 
$g=$ constante de gravedad

$B=$ ancho de la superficie plana (en el experimento es igual a $75 \mathrm{~mm}$ )

$\theta=$ ángulo del tanque o cuadrante (en el experimento es igual a $0^{\circ}$ )

$R_{1}=$ radio interior (en el experimento es igual a $100 \mathrm{~mm}$ )

$R_{2}=$ radio exterior (en el experimento es igual a 200 $\mathrm{mm}$ )

$R_{3}=$ brazo de palanca (en el experimento es igual a 200 $\mathrm{mm}$ )

$\rho g$ puede ser obtenido del Caso I, de tal manera que $h^{3}$ puede entonces graficarse en contra de $M+\rho g B h R_{2}^{2} / 2$.

\section{Fuerza hidrostática y su posición}

En este experimento, se han analizado dos casos. El Caso 1 está totalmente sumergido donde el nivel del agua $\left(h_{2}\right)$ es mayor que la altura del plano mientras que el Caso 2 está parcialmente sumergido donde el nivel del agua $\left(h_{1}\right)$ es menor que el plano como se muestra en la Figura 6.

\section{Plano completamente sumergido}

La Figura 7 muestra la distribución de la presión a lo largo del plano vertical cuando el nivel del agua es mayor que la altura del plano.

\section{Magnitud de la fuerza resultante total $F_{R}$}

$$
F_{R}=\int_{A} P d A=\int_{y=R_{1}-h_{2}}^{y=R_{2}-h_{2}} \rho g B y d y
$$

Sugerencia: Considerado el balance de torques debido a la fuerza $\mathbf{F}_{\mathbf{R}}$

Posición de $\mathbf{F}_{\mathbf{R}}$ sobre el eje $y$

$y_{c p}$ : es la coordenada $y$ del punto de acción de la fuerza $F_{R}$

$$
\begin{gathered}
y_{c p}=\frac{\int_{A_{\text {sumergida }}} y^{2} d A}{\int_{A_{\text {sumergida }}} y d A}=\frac{I_{x x}}{\int_{A_{\text {sumergida }}} y d A} \\
=\frac{A_{\text {sumergida }} y_{c}^{2}+I_{x x, c}}{A_{\text {sumergida }} y_{c}}=y_{c}+\frac{I_{x x, c}}{A_{\text {sumergida }} y_{c}}
\end{gathered}
$$

Donde:

$$
y_{c}=\frac{R_{2}+R_{1}}{2}+\left(R_{1}-h_{2}\right)
$$

$$
I_{x x, c}=\frac{1}{12} B\left(R_{2}-h_{1}\right)^{3}
$$

Sustituyendo las Ecs. (18) y (19) en la Ec. (17), resulta en:

$$
\begin{aligned}
& y_{c p}=\frac{R_{2}-R_{1}}{2}+\left(R_{1}-h_{2}\right) \\
& +\frac{B\left(R_{2}-R_{1}\right)^{3}}{12 A_{\text {sumergida }}\left(\left(\frac{R_{2}-R_{1}}{2}\right)+\left(R_{1}-h_{2}\right)\right)}
\end{aligned}
$$



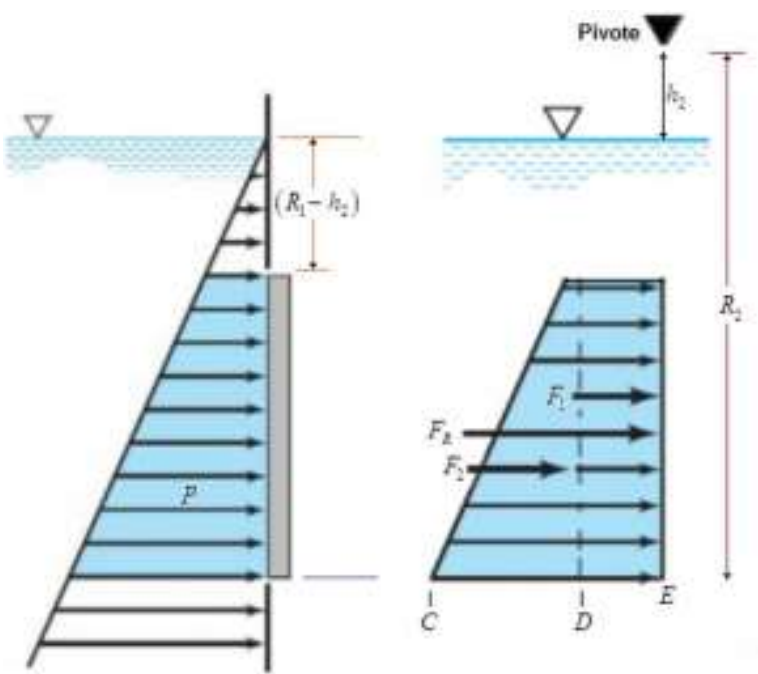

Figura 7. Esquema representativo cuando el nivel del agua es mayor que la altura del plano.

Fuente: Elaboración propia.

\section{Plano parcialmente sumergido}

La presión cambia linealmente y la fuerza hidrostática cambia a lo largo de la profundidad vertical $h$ como se muestra en la Figura 8.

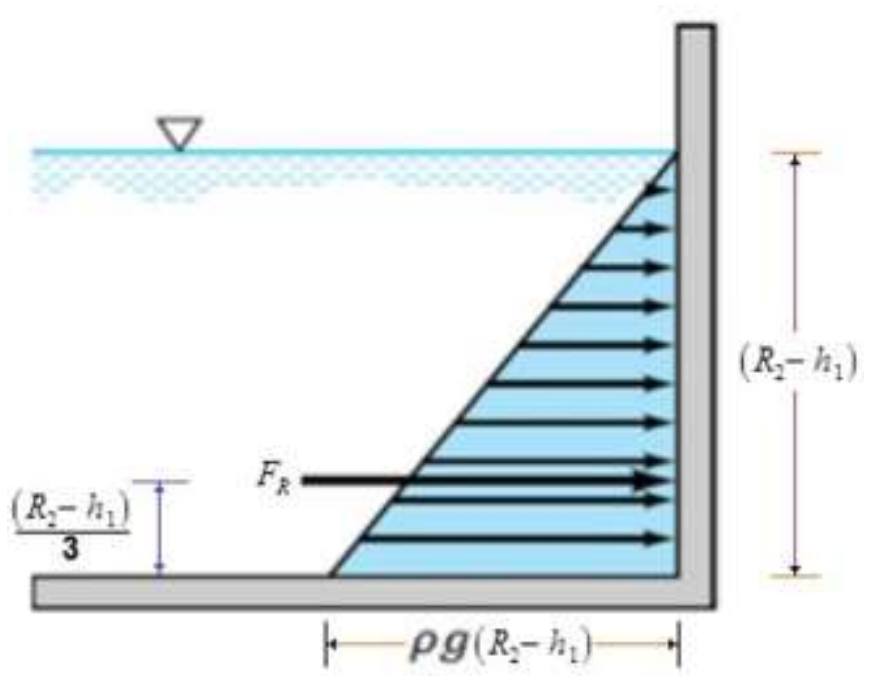

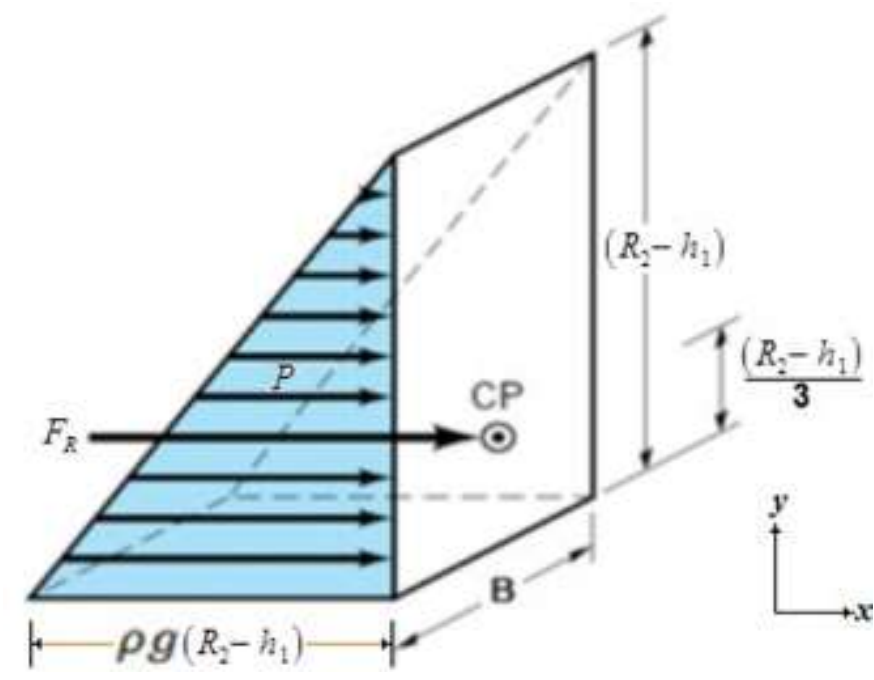

Figura 8. Esquema representativo cuando el nivel del agua es menor que la altura del plano.

Fuente: Elaboración propia.

La Figura 8, muestra la distribución de la presión a lo largo del plano vertical cuando el nivel del agua es menor que la altura del plano. Por otro lado, tenemos:

$$
\left(R_{2}-h_{1}\right)<\left(R_{2}-R_{1}\right)
$$

La fuerza diferencial actuando sobre el área diferencial $d A$ del plano es:

$$
d F=(\text { Presión }) \cdot(\text { Área })=(\rho g y) \cdot(d A)
$$

\section{La magnitud de la fuerza total resultante $F_{R}$}

$$
F_{R}=\int_{A} P \cdot d A=\int_{y=0}^{y=R_{2}-h_{1}} \rho g B y d y
$$

¿Dónde está el punto (ubicación) de acción de $F_{R}$ ? ¡iNo pasa a través del Centroide!! (¿Por qué?)

Sugerencia: Considerado el balance de torques debido a la fuerza $\mathbf{F}_{\mathbf{R}}$

Posición de $\mathbf{F}_{\mathbf{R}}$ sobre el eje $y$ 
$y_{c p}$ : es la coordenada $y$ del punto de acción de la fuerza

$F_{R}$

$$
y_{c p}=y_{c}+\frac{I_{x x, c}}{A y_{c}}
$$

donde:

$$
\begin{gathered}
y_{c}=\frac{R_{2}-h_{1}}{2} \\
I_{x x, c}=\frac{1}{12} B\left(R_{2}-h_{1}\right)^{3}
\end{gathered}
$$

Sustituyendo las Ecs. (25) y (26) en la Ec. (24), se tiene:

$$
y_{c p}=\frac{R_{2}-h_{1}}{2}+\frac{B\left(R_{2}-h_{1}\right)^{3}}{12 A_{\text {sumergida }}\left(\frac{R_{2}-h_{1}}{2}\right)}
$$

\section{Materiales y procedimiento}

Esta práctica permite que los estudiantes puedan medir el momento debido al empuje fluido (hidrostático) sobre un plano completamente o parcialmente sumergido. El plano funciona en posición vertical o inclinada (en ángulo). Los estudiantes luego comparan sus mediciones con el análisis teórico. El equipo centre of pressure apparatus modelo $\mathrm{H} 11$ (ver Figura 9) consiste en un panel vertical que sostiene un cuadrante de plástico claro, al que los estudiantes agregan el agua. El cuadrante tiene líneas grabadas para ayudar a los estudiantes a mantener el plano en una posición vertical o en ángulo. Los lados cilíndricos del cuadrante tienen su eje central coincidente con el eje de medición del momento. Por lo tanto, las presiones totales de fluido sobre estas superficies curvadas no ejercen ningún momento sobre este pivote. Por lo tanto, el momento es sólo debido a la presión del fluido en la superficie de prueba plana. Los estudiantes miden este momento usando pesos suspendidos de un brazo nivelado. Una escala en el panel del aparato, muestra el cabezal del agua. Para realizar experimentos, los estudiantes nivelan el aparato usando sus pies de nivelación y nivel de esencia (burbuja). Ellos deciden si probar un plano vertical o inclinado. A continuación, inicialmente equilibran el tanque del cuadrante con una de las suspensiones de peso y el tanque de ajuste más pequeño. Tienen los resultados, equilibrando pesos incrementales en el colgador, con cantidades conocidas de agua. Luego usan los resultados para calcular el momento equivalente de fuerza $(M)$ o empuje hidrostático. Los estudiantes observan la relación entre el momento y la altura del agua (h).

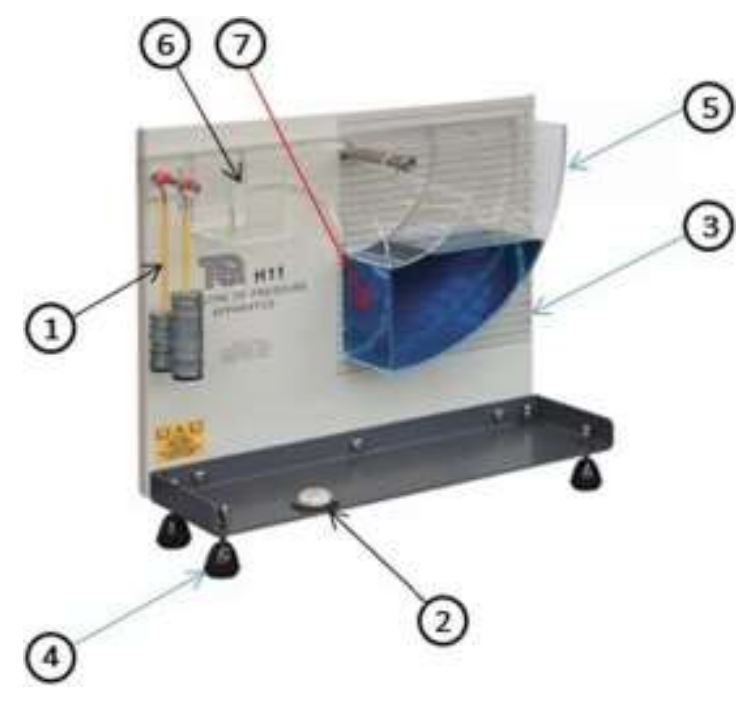

Figura 9. Equipo empleado para encontrar el centro de presión de una superficie totalmente sumergida 0 parcialmente. 1: Colgador de pesos incrementales; 2: Nivel de esencia (burbuja), 3: Escala; 4: Pies de nivelación; 5: Cuadrante de plástico claro; 6: Depósito de compensación; 7: Pared plana.

Fuente: Elaboración propia.

1. Polvo para preparar una tintura de agua no tóxica (ver Figura 10), que ayuda a los estudiantes a ver los niveles de agua más claramente. 


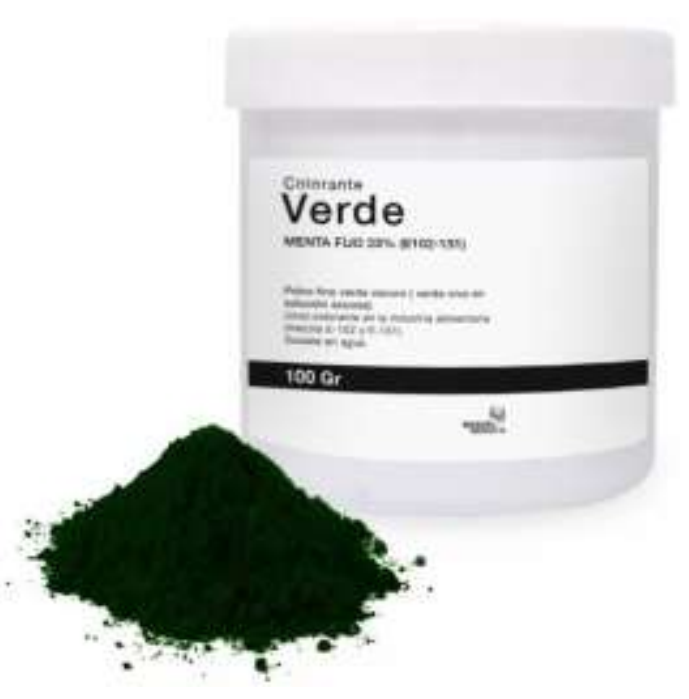

Figura 10. Polvo para preparar una tintura de agua. Fuente: Elaboración propia.

2. Jeringa para la adición precisa o la eliminación de pequeñas cantidades de agua (ver Figura 11).

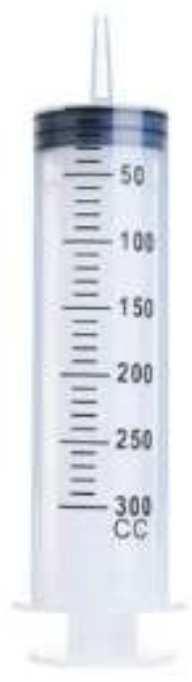

Figura 11. Jeringa.

Fuente: Elaboración propia.

3. Colgador de pesos (ver Figura 12).

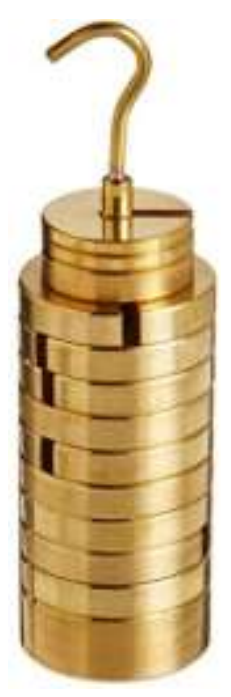

Figura 12. Colgador de pesos. Fuente: Elaboración propia.

Equipo de protección individual a utilizar:

- Bata blanca

- Botas de seguridad

- Guantes de protección térmica

- Gafas de protección

Realice el experimento siguiendo los siguientes pasos:

- Enganche un colgador de pesas (vacío) al soporte $y$ agregue agua al tanque de compensación hasta que el tanque esté nivelado $y$, por lo tanto, el plano sumergido sea vertical. La línea horizontal en la parte posterior del tanque debe alinearse con la línea de $0 \mathrm{~mm}$ del panel posterior. El colgador de pesas vacío tiene un peso de acabado de $10 \mathrm{~g}$. No es necesario grabar el peso o la cantidad de agua en el depósito de compensación, ya que son simplemente para equilibrar el tanque vacío a 0 grados.

- Agregue el segundo colgador de pesas con un peso de $10 \mathrm{~g}$, dando un peso total de $20 \mathrm{~g}$ (el colgador de pesas es de $10 \mathrm{~g}$ ).

- Vierta el agua coloreada en el tanque cuadrante hasta que vuelva a $0^{\circ}$. Registre el peso y la lectura de la altura del agua $(h)$ con respecto a 0 $\mathrm{mm}$. 
- Continúe aumentando el peso en incrementos de $20 \mathrm{~g}$, mientras agrega agua hasta que el tanque se nivela. Deténgase cuando el nivel del agua $(h)$ alcance los $0 \mathrm{~mm}$ o haya utilizado todos los pesos. Registre el peso y el nivel en cada incremento en la Tabla 1.

\section{Resultados}

Las lecturas se pueden tabular en la forma descrita en la Tabla 1 y los resultados se pueden calcular de acuerdo con la teoría.

Tabla 1. Lecturas de masa y profundidad del agua. Fuente: Elaboración propia

\begin{tabular}{|c|c|c|c|}
\hline \multicolumn{2}{|c|}{ Prueba } & $\begin{array}{c}\text { Masa, } m \\
(\mathrm{~kg})\end{array}$ & $\begin{array}{c}\text { Protundidad, } h \\
(\mathrm{~m})\end{array}$ \\
\hline \multirow{4}{*}{$\begin{array}{c}\text { Parcialmente } \\
\text { sumergido }\end{array}$} & 1 & & \\
\cline { 2 - 4 } & 2 & & \\
\cline { 2 - 4 } & 3 & & \\
\cline { 2 - 4 } & 4 & & \\
\hline \multirow{3}{*}{$\begin{array}{c}\text { Totaimente } \\
\text { sumergido }\end{array}$} & 5 & & \\
\cline { 2 - 4 } & 7 & & \\
\cline { 2 - 4 } & 8 & & \\
\cline { 2 - 4 } & 9 & & \\
\cline { 2 - 4 } & 10 & & \\
\cline { 2 - 4 } & 9 & & \\
\hline \hline
\end{tabular}

\section{Cálculos}

Calcule lo siguiente para los cuadrantes sumergidos parcial y totalmente, regístrelos en la Tabla 2 de resultados:

Tabla 2. Lecturas y cálculos para los cuadrantes sumergidos parcialmente y totalmente.

Fuente: Elaboración propia

\begin{tabular}{|c|c|c|c|c|c|}
\hline $\begin{array}{l}\text { No } \\
\text { Prueba }\end{array}$ & $\begin{array}{l}\text { Masa, mt } \\
(\mathrm{kg})\end{array}$ & $\begin{array}{c}\text { Profundidad, } \\
\text { h. } \\
\text { (m) }\end{array}$ & $\begin{array}{c}\text { Fuerza } \\
\text { hidrostática } \\
\text { Fa } \\
\text { (N) }\end{array}$ & \begin{tabular}{|c|} 
Punto \\
(ubicación \\
de acción \\
de Fr \\
(Teónico) \\
(m)
\end{tabular} & $\begin{array}{c}\text { Punto } \\
\text { (ubicación) de } \\
\text { accoón de Fr } \\
\text { (Experimental) } \\
\text { (m) }\end{array}$ \\
\hline 1 & & & & & \\
\hline 2 & & & & & \\
\hline 3 & & & & & \\
\hline 4 & & & & & \\
\hline 5 & & & & & \\
\hline 6 & & & & & \\
\hline 7 & & & & & \\
\hline 8 & & & & & \\
\hline 9 & & & & & \\
\hline 10 & & & & & \\
\hline
\end{tabular}

\section{Conclusiones}

Se estudió la fuerza hidrostática en una superficie plana vertical total o parcialmente sumergida, a través de la definición fundamental de presión y se hizo una comparación entre ambas configuraciones de llenado teórico y práctico.

\section{Agradecimientos}

El trabajo descrito en el presente artículo fue financiado por la beca otorgada por PROMEP. Asimismo, le agradezco al Dr. Jorge Zuno Silva, quien es Director de la Escuela Superior de Ciudad Sahagún-UAEH, México, por todas las facilidades para concluir este trabajo.

\section{Referencias}

[1] Çengel, Y. A., \& Cimbala, J. M. (2018). Mecánica de fluidos: Fundamentos y aplicaciones / Yunus A. Çengel y John M. Cimbala. México D.F.: McGraw Hill

[2] White, F., (2018), Mecánica de fluidos, Ed. McGraw Hill

[3] Barrero Ripoll, A., Pérez-Saborid Sánchez-Pastor, M., (2005) Fundamentos y aplicaciones de la Mecánica de Fluidos, Ed. McGraw Hill

[4] Bauman R. P, (2000), An alternative derivation of Bernoulli's principle. Am. J. Phys. 68 (3), p. 288-289

[5] Walker J., (1998), The hydrostatic paradox: simple geometries, explicit calculations. The Physics Teacher, Vol. 36, pp. 378-379.

[6] Wilson A., (1995), The hydrostatic paradox. The Physics Teacher, Vol. 33 , pp. 538-539.

[7] Gaffney C., (2000), The hydrostatics of trapped bubbles in fluids. The Physics Teacher, vol 38, pp. 458-460.

[8] Franco A., (1982), Analogías Físicas (Mecánica, Electricidad, Fluidos). Documentación E.I. (Enseñanzas Integradas). Vol 6 (3), págs. 63-69

[9] Doltz M, Hernández M J, Delegido J, Casanovas A., (2006), A laboratory experiment on inferring Poiseuille's law for undergraduate students. Eur. J. Phys. 27; 1083-1089

[10] Mott, R.: «Mecánica de fluidos aplicada». Ed. Prentice Hall 